Jour. Vac. Sci. Techn., May 1997

\title{
The role of zero-point effects in catalytic reactions involving hydrogen
}

\author{
Axel Gross and Matthias Scheffler \\ Fritz-Haber-Institut der Max-Planck-Gesellschaft, Faradayweg 4-6, D-14195 Berlin-Dahlem, Germany
}

\begin{abstract}
According to the Heisenberg uncertainty principle of quantum mechanics, particles which are localized in space by a bounding potential must have a finite distribution of momenta. This leads, even in the lowest-possible energy state, to vibrations, and thus, to the so-called zero-point energy. For chemically bound hydrogen the zero-point energy can be quite substantial. For example, for a free $\mathrm{H}_{2}$ molecule it is $0.26 \mathrm{eV}$, a significant value in the realm of chemistry, where often an energy of the order of $0.1 \mathrm{eV} /$ atom (or $2.3 \mathrm{kcal} / \mathrm{mol}$ ) decides whether or not a chemical reaction takes place with an appreciable rate. Yet, in many theoretical studies the dynamics of chemical reactions involving hydrogen has been treated classically or quasi-classically, assuming that the quantum mechanical nature of $\mathrm{H}$ nuclei, i.e. the zero-point effects, will not strongly affect the relevant physical or chemical properties. In this paper we show that this assumption is not justified. We will demonstrate that for very basic and fundamental catalytic-reaction steps, namely the dissociative adsorption of molecular hydrogen at transition metal surfaces and its timereverse process, the associative desorption, zero-point effects can not only quantitatively but even qualitatively affect the chemical processes and rates. Our calculations (treating electrons as well as $\mathrm{H}$ nuclei quantum-mechanically) establish the importance of additional zero-point effects generated by the $\mathrm{H}_{2}$-surface interaction and how energy of the $\mathrm{H}-\mathrm{H}$ stretch vibration is transferred into those and vice versa.
\end{abstract}

Recently density functional theory calculations of the important diversity of pathways for chemical reactions at extended metal surfaces became possible [1] 내. Still, these calculated high-dimensional $a b$ initio potentialenergy surfaces (PES), which fully determine the energetics of the nuclei, are too complex that their consequences on the chemical reaction dynamics were obvious. It is worth to stress at this point that these calculations revealed that the high-dimensional nature of the PES can be very important, i.e., a restriction to just one reaction pathway is often not sufficient [4, 5]. In order to analyze and understand the catalytic dissociation it is important and possibly even necessary to combine the information about the high-dimensional PES of the chemical reaction with a subsequent calculation of the dynamics of the nuclei, moving along this PES. Typically this dynamics is described applying the laws of classical mechanics, the main motivation of this approach being its simplicity. Some studies of dissociative adsorption (and associative desorption) of $\mathrm{H}_{2}$, which is the topic we concentrate on in this paper, had addressed the issue of comparing classical and quantum dynamics [6 9], but in these studies only up to four degrees of freedom of the hydrogen molecule were treated quantum mechanically; obviously, the dynamics of two nuclei is determined by six coordinates. In the following we will restrict our discussion to the dynamics of dissociative adsorption. However, our findings about the importance of zero-point effects are equally valid for the associative desorption since the processes of dissociative adsorption and associative desorption are related by the principle of microscopic reversibility.

In this paper we will show that a quantum-mechanical, six-dimensional treatment of the nuclei exposes several interesting and important properties. Such calculations became possible only recently [5], and as a first example we studied the dissociative adsorption of $\mathrm{H}_{2}$ at the (100) surface of palladium (for the geometry of the (100) surface see the inset of Fig. 1). The employed high-dimensional PES was derived from density functional theory calculations [4] which revealed that it is strongly corrugated and that many activated and only few non-activated paths towards dissociative adsorption exist. Figure 11 shows a cut through this PES, which displays one of the few non-activated pathways towards dissociated adsorption. In this example the $\mathrm{H}_{2}$ molecule approaches with an orientation parallel to the surface and its center of mass at the bridge position (see inset of Fig. 11).

The six-dimensional quantum dynamical calculations show that the probability for dissociative adsorption of $\mathrm{H}_{2}$ at the clean $\mathrm{Pd}(100)$ is very high at low kinetic translational energies of the impinging molecules (up to $70 \%$ ), and that it decreases substantially when the kinetic translational energy is increased (to $25 \%$ at $\left.E_{i}=0.1 \mathrm{eV}\right)$. This result is displayed as the full curve in Fig. 2; it is in fact in good agreement with experimental findings [10]. We note that thermal energies are in the just noted range, i.e. typically $E_{i} \leq 0.1 \mathrm{eV}$. The analysis of these results revealed that the decrease of the sticking probability with increasing kinetic translational energy is not due to a precursor-mediated mechanism, as was commonly believed, but due to dynamical steering [5]: If the impinging molecule is slow the attractive forces of the PES steer it towards a favorable (non-activated) pathway, even if the initial conditions were pointing towards an unfavorable direction. However, if the molecule is fast, its inertia hinders its reorientation. Such a molecule will then probably experience an energy barrier and thus will be reflected. Of course, a very fast molecule will simply overrun energy barriers. Another result of the calculations was that the dissociation should be hindered if low 


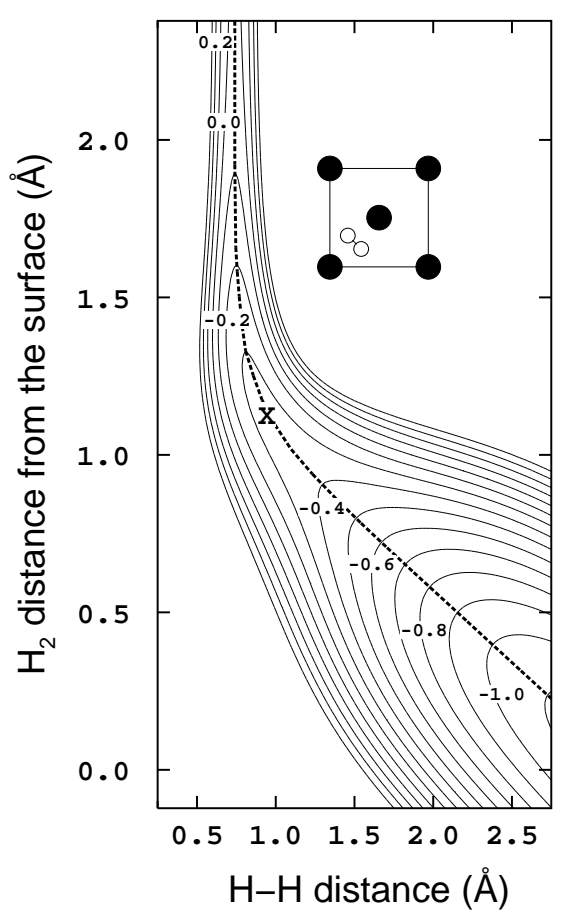

FIG. 1. Contour plot of the PES along a two-dimensional cut through the six-dimensional coordinate space of $\mathrm{H}_{2} / \mathrm{Pd}(100)$. The inset shows the orientation of the molecular axis and the lateral $\mathrm{H}_{2}$ center-of-mass coordinates. The coordinates in the figure are the $\mathrm{H}_{2}$ center-of-mass distance from the surface $Z$ and the $\mathrm{H}-\mathrm{H}$ interatomic distance $d$. The dashed line is the optimum reaction path. Energies are in $\mathrm{eV}$ per $\mathrm{H}_{2}$ molecule. The contour spacing is $0.1 \mathrm{eV}$.

energy molecules are rotationally excited, the effect also being dependent on the molecular orientation, and this prediction has been indeed confirmed by recent experiments 11,12 .

In this paper we will discuss the importance of quantum effects in dissociation and adsorption. At first we note that calculated $\mathrm{H}_{2}$ sticking probability exhibits a strong oscillatory structure as a function of the incident energy (see the dashed line in Fig. 2). These oscillations are a direct consequence of the quantum nature of $\mathrm{H}_{2}$ scattering. In a simplified description, i.e., neglecting rotational and vibrational degrees of freedom of $\mathrm{H}_{2}$, elastic scattering at a periodic surface gives rise to reflected beams, travelling in directions $\left(\mathbf{k}_{\|}+\right.$ $\left.\mathbf{g},-\sqrt{k_{z}^{2}-2 \mathbf{k}_{\|} \cdot \mathbf{g}-\mathbf{g}^{2}}\right)$. Here $\left(\mathbf{k}_{\|}, k_{z}\right)$ is the wave vector of the incident $\mathrm{H}_{2}$ beam and $\mathbf{g}$ is a two-dimensional reciprocal lattice vector of the surface. The condition for emerging beams is that the argument under the square root is positive. Just before a new beam can emerge, it is already built up though it remains confined to the surface. Thus, this beam can not (yet) be observed directly, but, as it is coherent with the other beams, these others will exhibit sharp resonance structures. In ad-

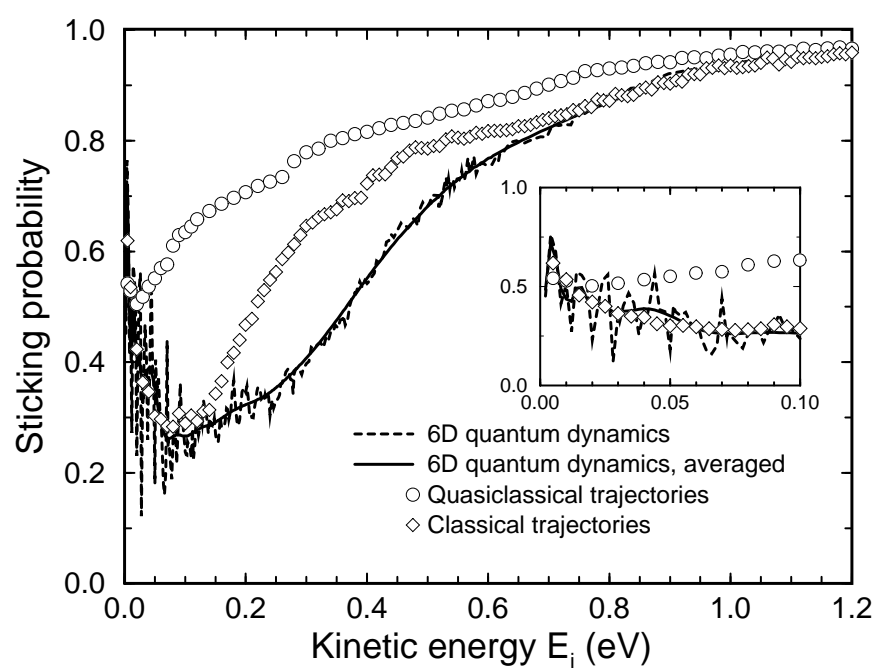

FIG. 2. Probability for dissociative adsorption versus kinetic translational energy for a $\mathrm{H}_{2}$ beam under normal incidence on a clean $\operatorname{Pd}(100)$ surface. The six-dimensional quantum dynamical results (solid and dashed line) are for molecules initially in the vibrational and rotational ground state. For the full line an energy spread typical for beam experiments has been assumed. Diamonds show results of a classical treatment of the $\mathrm{H}$ nuclei (initially non-vibrating molecules) and circles correspond to a quasi-classical treatment, i.e. the molecules vibrate initially with an energy of $0.26 \mathrm{eV}$, which is the zero-point energy of $\mathrm{H}_{2}$. The inset shows an enlargement of the results at low-energies.

dition, oscillations can also be caused by selective adsorption resonances 13 . These structures are known in $\mathrm{He}$ and $\mathrm{H}_{2}$ scattering since the 1930s [14 and in electron scattering [15, 16], however, for the particular system $\mathrm{H}_{2} / \mathrm{Pd}(100)$ they have not been observed yet. It is obvious that an experimental verification requires special care as the strengths and energies of the oscillatory structures depend sensitively on the initial conditions, and on the quality of the surface. For example, surface imperfections (e.g. adatoms and steps) will destroy the mentioned coherence and thus suppress the oscillations.

In previous experiments [10] the molecular beam was not strictly mono-energetic. By taking the experimental energy spread into account, $\Delta E / E_{i}=0.2 \sqrt{10}$, the oscillations of the quantum-mechanical study are smoothed out and the dashed line in Fig. 2 is turned into the solid one.

We have now complemented the six-dimensional quantum dynamical study by classical molecular dynamics simulations using exactly the same PES. This allows for the first time a thorough comparison of classical and quantum dynamics of $\mathrm{H}_{2}$ scattering, dissociation, and adsorption.

A common approach in classical calculations aiming at taking some quantum effects of the $\mathrm{H}_{2}$ molecule into account is to give the hydrogen molecules initially a 
vibrational energy which equals the $\mathrm{H}_{2}$ zero-point energy 6, 67, 17, 18. This is called a quasi-classical approach. The open circles in Fig. 2 display the corresponding results. It is quite obvious that they are quantitatively and even qualitatively at variance with the correct, i.e. quantum-dynamical, result (dashed curve in Fig. 2). Firstly, the quasi-classical calculations do not show any oscillatory structure. Secondly, they do not exhibit any pronounced initial decrease with increasing kinetic translational energy, i.e. steering is absent in the quasi-classical calculations. Only at very high energies, where quantum effects play a minor role, quantum and quasi-classical trajectory calculations get into agreement.

The diamonds in Fig. 2 correspond to a strict classical treatment where the $\mathrm{H}_{2}$ molecules have initially no vibrational energy. It is interesting to note that these calculations agree much better with the quantum-mechanical results than the quasi-classical one. Again, the quantum oscillations are obviously absent, but now the steering effect is in operation. The absence of the oscillatory structure in the classical and quasi-classical calculations is caused by the fact that the coherence and interference of molecular beams is not present in classical mechanics.

We will now show that further quantum effects, namely the generation of additional zero-point energies, lead to the qualitiative difference between the quasi-classical and the averaged quantum dynamical calculations. The interaction of the $\mathrm{H}_{2}$ molecule with the surface gives rise to these additional zero-point energies (due to hindered rotations and hindered surface diffusion) and this energy, obviously, needs to be taken from that of the incident beam. For the ease of the following discussion we will assume that the approaching molecule travels along the optimum reaction pathway (full line in Fig. 1). Let us at first describe the correct, i.e. quantum-mechanical, results. When the molecule gets close to the surface, the energy of its stretch vibrations is lowered, because when the $\mathrm{H}$-surface bond starts to build up, the $\mathrm{H}-\mathrm{H}$ bond strength will at the same time soften. Since the vibrational frequency is high, the change of the frequency during one oscillation is small compared to the frequency, i.e.

$$
t_{\mathrm{vib}} \cdot \frac{d \omega_{\mathrm{vib}}}{d t} \ll \omega_{\mathrm{vib}}
$$

This suggests that the $\mathrm{H}_{2}$ vibration follows the molecular center of mass motion adiabatically, which is indeed confirmed within a very good approximation by the quantum mechanical [19] as well as by the classical calculations. Interestingly, the change in zero-point energy is not transferred to translational motion. Thus, in contrast to common believe, it cannot be utilized to overcome energy barriers. The reason is that at the point where the $\mathrm{H}-\mathrm{H}$ stretch-frequency is lowered, i.e. where the $\mathrm{H}-\mathrm{H}$ bond is already weakened, also a noticeable H-surface bond has already started to develop, as

\begin{tabular}{|c|c|}
\hline \hline mode & zero-point energy $(\mathrm{eV})$ \\
\hline H-H vibration & 0.126 \\
polar rotation & 0.084 \\
azimuthal rotation & 0.033 \\
translation perpendicular to bridge & 0.051 \\
translation parallel to bridge & 0.056 \\
\hline sum & 0.350 \\
\hline \hline
\end{tabular}

TABLE I. Zero-point energies of the $\mathrm{H}_{2}$ molecule at the point where the sum of all zero-point energies has its maximum (marked by $\mathrm{X}$ in Fig. 1). The $\mathrm{H}_{2}$ center-of mass is above the bridge position between two adjacent $\mathrm{Pd}$ atoms (see inset of Fig. 11). The energies are given per $\mathrm{H}_{2}$ molecule.

was noted already above. In other words, close to the surface the PES is strongly corrugated. This leads to the building up of zero-point energies in the degrees of freedom of the hydrogen molecule perpendicular to the optimum reaction path. In table fill these calculated zero-point energies are listed at the point where their sum has its maximum. This geometry is indicated in Fig. 1 by the cross. Although the zero-point energy of the stretch vibrations is significantly lowered, this lowering is over-compensated by the zero-point energies in the other modes so that the sum of all zero-point energies, $0.35 \mathrm{eV}$ per $\mathrm{H}-\mathrm{H}$ pair is even larger than the $\mathrm{H}_{2}$ gasphase value of $0.26 \mathrm{eV}$. That the sum of all zero-points energies in the case of hydrogen dissociative adsorption is approximately equal or even higher than in the gasphase has also been found in total-energy calculations for $\mathrm{H}_{2} / \mathrm{Al}(110)$ 20 and $\mathrm{H}_{2} / \mathrm{Si}(100)$ [21, where values of $0.25 \mathrm{eV} /$ molecule and $0.29 \mathrm{eV} /$ molecule have been determined at the top of the barrier to dissociative adsorption.

Obviously, in classical or quasi-classical calculations the concept of zero-point vibrations does not exist. Therefore in the quasi-classical calculations the vibrational energy can be almost fully transferred to the translational energy which enables the molecule to traverse energy barriers with a height larger than its initial kinetic translational energy. This is actually the reason why the steering effect is absent in the quasi-classical results (open dots in Fig. 2): These vibrating molecules do not experience a strong energy barrier because the vibrational energy is efficiently transferred to translational energy.

The analysis of the zero-point energies along the optimum reaction pathway (see Fig. 1) teaches us that the sum of all zero-point energies is roughly constant during the dissociation. This approximate "constant of motion" can be simulated in classical trajectory calculations by ignoring the zero-point energies all along the way. As mentioned above, this strictly classical treatment (see the diamonds in Fig. 2) gives results in remarkable agreement 
with the averaged quantum dynamical results at low and high energies. The disagreement in the medium energy regime could perhaps be due to the fact that the sum of the zero-point energies is not really constant but becomes larger than the gas-phase zero-point energy so that the quantum mechanical sticking probability is lower due to the effectively higher barriers.

Our results clearly show that for kinetic translational energies between 0.15 and $0.6 \mathrm{eV}$ the probability for dissociative adsorption is reduced by quantum effects by $50 \%$ compared to the quasi-classical result. Thus, in contrast to the common belief, quantum effects in the dynamics of $\mathrm{H}_{2}$ chemical reactions at surfaces are clearly not negligible.

An influence of the zero-point energies in traversing barrier regions has also been found in the comparison of three-dimensional quantum and classical calculations of the dissociative adsorption of $\mathrm{H}_{2} / \mathrm{Cu}(111)$ [9]. However, since these calculations were restricted to low dimensions, the difference between quantum and classical results was much less pronounced because the sum of the zero-point energies was smaller. From the above discussion it is clear that a proper theoretical description of the role of zero-point effects requires a high-dimensional quantummechanical treatment of the nuclear motion.

The problem of a proper treatment of zero-point energies in quasiclassical trajectory calculations is wellknown, especially in the gas-phase community [17,18. One possible way to deal with this problem is the reduced dimensionality treatment in the vibrationally adiabatic approximation (for a overview see Ref. [22]). In this approach a small number of degrees of freedom is treated dynamically while the remaining degrees of freedom are taken into account by adding the sum of their zero-point energies to the potential along the reaction path. Another more elaborate approach is to constrain the energy in each vibrational mode to be greater than its zero-point value [17, 18 .

In our purely classical approach we ignore zero-point energies all along the reaction path. But this approach is actually in the spirit of the vibrationally adiabatic approximation. It effectively takes the zero-point energies into account through a shift of the potential along the reaction path corresponding to the sum of all zero-point energies. This shift, however, is constant along the reaction path. Moreover, we still keep the full dimensionality of the problem by explicitly treating all degrees of freedom dynamically. This is indeed essential since for example the steering effect is absent in a low-dimensional treatment of the $\mathrm{H}_{2} / \mathrm{Pd}(100)$ system [23] (see also the discussion below).

In order to illustrate some of the dynamical effects discussed above we have plotted in Figs. 3 and 1 some typical trajectories of the classical molecular dynamics calculations. The upper panel shows snapshots of trajectories of the impinging $\mathrm{H}_{2}$ molecules while the lower panel dis-

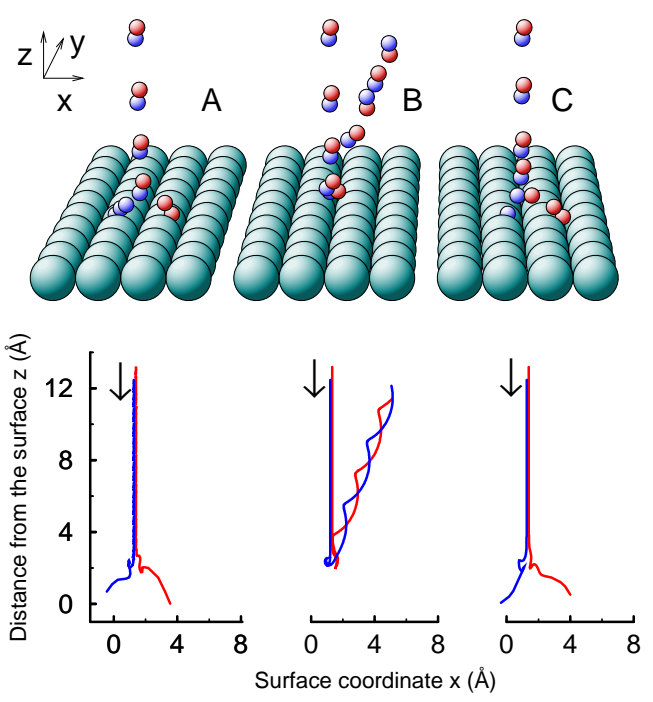

FIG. 3. Classical trajectories of a hydrogen molecule impinging on a $\operatorname{Pd}(100)$ surface. The initial conditions are chosen in such a way that the trajectories are restricted to the $x z$-plane. Upper panel: snapshots of the trajectories; lower panel: trajectories in the $x z$-plane. Trajectory A: quasi-classical trajectory with an initial kinetic translational energy of $0.12 \mathrm{eV}$. Trajectory B: same initial conditions as in trajectory $\mathrm{A}$, except that the molecule is initially non-vibrating. Trajectory C: same initial conditions as in trajectory $\mathrm{B}$, except that the molecule has a lower kinetic translational energy of $0.01 \mathrm{eV}$.

plays the whole trajectories. The initial conditions are chosen in such a way that the trajectories are restricted to a $x z$-plane. Trajectories A and B in Fig. 3 illustrate the difference between quasi-classical and classical calculations. They have identical initial conditions with a kinetic translational energy of $0.12 \mathrm{eV}$ except for the fact that in case A the molecule initially vibrates with an energy corresponding to the zero-point energy while in case $\mathrm{B}$ the molecule is initially non-vibrating (due to the small amplitude of $0.12 \AA$ the vibrations are hardly perceivable on the scale of the figure). For the chosen initial conditions of the impinging molecules there is an energy barrier hindereing the dissociative adsorption, and the initial translational energy is not large enough for the molecule to overcome this. Thus, the molecule is scattered back at the repulsive potential (trajectory $\mathrm{B}$ ), whereas if the initial vibrational zero-point energy is transferred to the translation due to the adiabaticity of the vibrations (trajectory $\mathrm{A}$ ), the molecule can directly dissociate.

Finally, trajectory $\mathrm{C}$ illustrates the steering effect [5, 8$]$. The initial conditions are as in trajectory $\mathrm{B}$, but now the molecule has a much lower kinetic translational energy, $E_{i}=0.01 \mathrm{eV}$. The molecule is so slow that the attractive forces can reorient the molecule so that it can follow a non-activated path towards dissociative adsorption. We like to emphasize that steering works in all geometry parameters of the nuclei and becomes more important when 
the configuration space is high-dimensional - in a onedimensional description (just restricting the reaction to "the optimum" reaction pathway) or a two-dimensional description (restricting the dynamics to only one elbow [23]), the steering effect is absent.

Trajectory $\mathrm{C}$ in Fig. 3 represents a classical illustration of steering. We note that this picture also applies when the quantum nature of $\mathrm{H}_{2}$ molecules is considered, and we now briefly address the quantum mechanical picture. Let us assume that the incident $\mathrm{H}_{2}$ molecule is in the rotational ground state, $j_{i}=0$ (the description for rotationally excited states is analogous). The $j_{i}=0$ state refers to an isotropic distribution of molecular orientation. Thus, the molecule is not rotating, but when the orientations of different molecules in the beam were measured, one would find all orientations with the same probability. In other words, the probability that a molecule impinges with the favorable orientation noted in Fig. 1 is very low. For molecules with low kinetic energy the steering effect will align all molecules, and trajectory $\mathrm{C}$ shows one example, which acts on the molecular polar coordinate $\theta$. Equivalent processes take place in the azimuthal $\phi$ and the $(X, Y)$ coordinates. Also with respect to the $(X, Y)$ coordinates of the $\mathrm{H}_{2}$ molecule we note their meaning in (basic) quantum mechanics: A plane wave of hydrogen molecules representing a molecular beam impinging under normal incidence on a surface has no $(X, Y)$ dependence in the gas-phase. This means that the probability to find a molecule at any given point point in a $(X, Y)$ plane far away from the surface is the same. This brief reminder to the concepts of quantum mechanics highlights also the big advantage of quantum dynamics in comparison to molecular dynamics. A quantum dynamical approach includes the ensemble of all $\mathrm{H}_{2}$ orientations $(\theta, \phi)$ and all $(X, Y)$ points of impact automatically. In a molecular dynamics calculations the same ensemble has to be built up step by step, i.e. by performing thousands of molecular dynamics runs, namely one run for each choice of $(X, Y, \theta, \phi)$.

The trajectory in Fig. 4 finally indicates a process which in a quantum-mechanical system will give rise to an oscillatory structure (see Fig. 2). The initial conditions are as in the trajectory of Fig. $3 \mathrm{C}$, only the impact point in the surface unit cell and the inital polar orientation are slightly changed. The snapshots of the trajectory in the upper panel are taken approximately every 150 fs. They are consecutively numbered so that the dynamics can be roughly followed. Due to the strong anisotropy and the corrugation of the potential the translational energy perpendicular to the surface is efficiently transferred to rotational and kinetic energy parallel to the surface. Hence the molecule is temporarily trapped; in this case the molecule spends more than 5 ps in front of the surface before it is dissociated. Note that this trapping does not occur because of energy transfer to the surface but because of the energy redistribution among the $\mathrm{H}_{2}$ de-

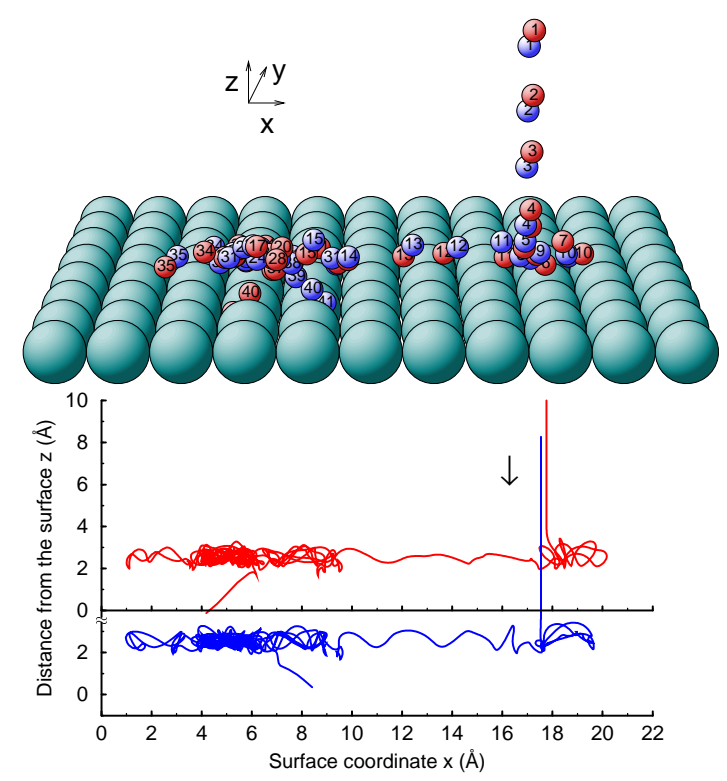

FIG. 4. Classical trajectory of a hydrogen molecule impinging on a $\operatorname{Pd}(100)$ surface. The initial kinetic energy is $0.01 \mathrm{eV}$, the initial conditions are only slightly different from those of Fig. 3C (see text). Upper panel: snapshots of the trajectories which are taken approximately every 150 fs and consecutively numbered. Lower panel: trajectories in the $x z$-plane; for the sake of clarity the trajectories are shifted in the $z$-direction with respect to each other.

grees of freedom. Quantum mechanically the trapped molecules correspond to metastable states at the surface which only exist for certain discrete energies; in addition, only discrete values for the energy and momentum transfer are allowed. Thus only for particular initial energies the dynamical trapping into these so-called selective adsorption resonances is possible [13,14. As mentioned in the discussion of Fig. 2, these resonances in addition to the opening up of scattering channels lead to the sharp peaks in the quantum-mechanical sticking probability.

In conclusion, we have shown that there are large differences between classical and quantum dynamics of hydrogen. The classical results do not show any oscillatory structure which in the quantum dynamics is due to the opening up of new scattering channels and resonances. Even more remarkable, zero-point effects can cause substantial deviations between classical and averaged quantum dynamical calculations. A corrugated and anisotropic potential energy surface leads to the building up of zero-point energies which effectively increase the minimum potential in the quantum calculations. This can change the dynamics of chemical reactions not only quantitatively, but also qualitatively, and thus strongly affects the rate constants of catalytic reactions involving hydrogen. These zero-point effects will also be relevant for other studies of hydrogen dynamics in corrugated potentials, like for example in hydrogen diffusion on surfaces or in the bulk. If, however, the sum of the zero-point en- 
ergies is approximately constant during the traversing of regions with energy barriers, which for hydrogen dissociation on surfaces seems to be the case for a wide class of systems, the discrepancies between classical and averaged quantum dynamics can be diminished by neglecting zero-point energies in the initial conditions.

[1] B. Hammer, M. Scheffler, K.W. Jacobsen, and J.K. Nørskov, Phys. Rev. Lett. 731400 (1994).

[2] J.A. White, D.M. Bird, M.C. Payne, and I. Stich, Phys. Rev. Lett. 731404 (1994).

[3] B. Hammer and M. Scheffler, Phys. Rev. Lett. 74, 3487 (1995).

[4] S. Wilke and M. Scheffler, Surf. Sci. 329, L605 (1995); Phys. Rev. B 53, 4926 (1996).

[5] A. Gross, S. Wilke, and M. Scheffler, Phys. Rev. Lett. 75, 2718 (1995).

[6] C. Engdahl and U. Nielsen, J. Chem. Phys. 98, 4223 (1993).

[7] C. Chiang and B. Jackson, J. Chem. Phys. 87, 549 (1987).

[8] M. Kay, G.R. Darling, S. Holloway, J.A. White, and D.M. Bird, Chem. Phys. Lett. 245, 311 (1995).

[9] A.D. Kinnersley, G.R. Darling, S. Holloway, and B. Hammer, Surf. Sci. 364, 219 (1996).

[10] K. D. Rendulic, G. Anger, and A. Winkler, Surf. Sci. 208, 404 (1989).

[11] M. Beutl, M. Riedler, and K.D. Rendulic, Chem. Phys. Lett. 247, 249 (1995)

[12] D. Wetzig, R. Dopheide, M. Rutkowski, R. David, and H. Zacharias, Phys. Rev. Lett. 76, 463 (1996).

[13] G.R. Darling and S. Holloway, J. Chem. Phys. 93 (1990) 9145.

[14] I. Esterman and O. Stern, Z. Phys. 61, 95 (1930); R. Frisch and O. Stern, Z. Phys. 84, 430 (1933).

[15] E. G. McRae, Rev. Mod. Phys. 51, 541 (1979).

[16] J. B. Pendry, Low energy electron diffraction, Academic Press, London (1974), p. 112.

[17] J.M. Bowman. B. Gazdy, and Q. Sun, J. Chem. Phys. 91, 2859 (1989).

[18] W. H. Miller, W. L. Hase, and C. L. Darling, J. Chem. Phys. 91, 2863 (1989).

[19] A. Gross and M. Scheffler, Chem. Phys. Lett. 256, 417 (1996).

[20] B. Hammer, K.W. Jacobsen and J.K. Nørskov, Phys. Rev. Lett. 69, 1971 (1992).

[21] P. Kratzer, B. Hammer, and J.K. Nørskov, Chem. Phys. Lett. 229, 645 (1994); Phys. Rev. B 51, 13432 (1995).

[22] The Theory of Chemical Reactions Dynamics, ed. by D. C. Clary, Reidel, Dordrecht, 1986.
[23] G. R. Darling and S. Holloway, Surf. Sci. 268, L305 (1992). 